\title{
Simplified disease activity changes in real-world practice: a nationwide observational study of seropositive rheumatoid arthritis patients with moderate-to-high disease activity
}

Kichul Shin ${ }^{1}$, Sung Soo Kim² ${ }^{2}$, Sang-Heon Lee 3 , Seung-Jae Hong ${ }^{4}$, Sung Jae Choi ${ }^{5}$, Jung-Yoon Choe ${ }^{6}$, Seung-Geun Lee 7 , Hoon-Suk Cha ${ }^{8}$, Eun Young Lee ${ }^{9}$, Sung-Hwan Park ${ }^{10}$, Jin-Wuk Hur ${ }^{11}$, Sung Soo $\mathrm{Na}^{12}$, Chang-Hee Suh ${ }^{13}$, Min Wook So ${ }^{14}$, Seung Won Choi ${ }^{15}$, Dong-Hyuk Sheen ${ }^{16}$, Won Park ${ }^{17}$, Shin-Seok Lee ${ }^{18}$, Wan Hee Ryu ${ }^{19}$, Jin Seok Kim ${ }^{20}$, Jung Soo Song ${ }^{21}$, Hye Soon Lee $^{22}$, Seong Ho Kim ${ }^{23}$, and Dae-Hyun Yoo ${ }^{24}$

Division of Rheumatology, ${ }^{1}$ Seoul Metropolitan Government Seoul National University Boramae Medical Center, Seoul; ${ }^{2}$ Gangneung Asan Hospital, University of Ulsan College of Medicine, Gangneung; ${ }^{3}$ Konkuk University Medical Center, Seoul; ${ }^{4}$ Kyung Hee University Hospital, Seoul; ${ }^{5}$ Korea University Ansan Hospital, Ansan; ${ }^{6}$ Daegu Catholic University Medical Center, Daegu; ${ }^{7}$ Pusan National University Hospital, Busan; ${ }^{8}$ Samsung Medical Center, Sungkyunkwan University School of Medicine, Seoul; ${ }^{9}$ Seoul National University Hospital, Seoul; ${ }^{10}$ College of Medicine, Seoul St. Mary's Hospital, The Catholic University of Korea, Seoul; ${ }^{11}$ Gangnam Eulji Hospital, Eulji University School of Medicine, Seoul; ${ }^{12}$ Soonchunhyang University Cheonan Hospital, Cheonan; ${ }^{13}$ Ajou University Hospital, Suwon; ${ }^{14}$ Pusan National University Yangsan Hospital, Yangsan; ${ }^{15}$ Ulsan University Hospital, Ulsan; ${ }^{16}$ Eulji University Hospital, Daejeon; ${ }^{17}$ Inha University Hospital, Incheon; ${ }^{18}$ Chonnam National University Hospital, Gwangju; ${ }^{19}$ Chonbuk National University Hospital, Jeonju; ${ }^{20}$ Jeju National University Hospital, Jeju; ${ }^{21}$ Chung-Ang University Hospital, Seoul; ${ }^{22}$ Hanyang University Guri Hospital, Guri; ${ }^{23}$ Inje University Haeundae Paik Hospital, Busan; ${ }^{24}$ Hanyang University Hospital for Rheumatic Diseases, Seoul, Korea

Background/Aims: The objective of this study was to compare changes in the simplified disease activity index (SDAI) between biologic (b) and conventional (c) disease-modifying antirheumatic drugs (DMARD) users with seropositive rheumatoid arthritis (RA) in daily clinical practice.

Methods: This was a nationwide multicenter observational study. Patients who had three or more active joint counts and abnormal inflammatory marker in blood test were enrolled. The selection of DMARDs was determined by the attending rheumatologist. Clinical parameters, laboratory findings, and Health Assessment Questionnaire (HAQ) scores were obtained at baseline and at 6 and 12 months. Serial SDAI changes and clinical remission rate at 6 and 12 months were assessed.

Results: A total of 850 patients participated in this study. The mean baseline SDAI score in bDMARD group was higher than that in cDMARD group $(32.08 \pm 12.98$ vs $25.69 \pm 10.97, p<0.0001)$. Mean change of SDAI at 12 months was -19.0 in the bDMARD group and -12.6 in the cDMARD group $(p<0.0001)$. Clinical remission rates at 12 months in bDMARD and cDMARD groups were $15.4 \%$ and $14.6 \%$, respectively. Patient global assessment and HAQ at 12 months were also significantly improved in both groups. Multivariate logistic regression showed that baseline HAQ score was the most notable factor associated with remission.

Conclusions: There was a significant reduction in SDAI within 12 months after receiving DMARDs in Korean seropositive RA patients irrespective of bDMARD or cDMARD use in real-world practice. Clinical remission was achieved in those with lower baseline HAQ scores.

Keywords: Arthritis, rheumatoid; Antirheumatic agents; Biological therapy

Received: April 12, 2018

Revised : July 5,2018

Accepted: July 5, 2018
Correspondence to Dae-Hyun Yoo, M.D.

Division of Rheumatology, Hanyang University Hospital for Rheumatic Diseases, 222-1 Wangsimni-ro, Seongdong-gu, Seoul 04763 , Korea Tel: +82-2-2290-9202, Fax: +82-2-2298-9231, E-mail: dhyoo@hanyang.ac.kr 


\section{INTRODUCTION}

Rheumatoid arthritis (RA) is a chronic progressive inflammatory condition that can lead to significant disability and joint pain $[1,2]$. In Korea, annual prevalence rate of RA estimated based on insurance claim data was $0.27 \%$ in 2009 [3]. The introduction of biologic therapies such as tumor necrosis factor alpha (TNF- $\alpha$ ) antagonists has dramatically improved treatment outcomes of severe RA. Biologic therapies now provide opportunities to target precise pathways within the immune system and inflammatory pathway, leading to better quality of life for RA patients in terms of disease activity control with reduced comorbidities and mortality.

Patients diagnosed with RA are first given conventional disease-modifying antirheumatic drugs (cDMARDs). However, the rate of using biologic DMARDs (bDMARDs) is rising rapidly [4,5]. Currently, numerous TNF- $\alpha$ antagonists are licensed for use in RA in Korea: infliximab, infliximab biosimilar, adalimumab, golimumab, TNF- $\alpha$ receptor fusion protein etanercept, and etanercept biosimilar. The efficacy and tolerability of TNF- $\alpha$ antagonists have been studied in randomized controlled clinical trials (RCTs) and observational studies [6-11]. A recent meta-analysis has also reported that these agents are superior to CDMARDs alone in patients with active disease [12]. However, data demonstrating disease activity changes by bDMARDs in parallel to cDMARDs in routine clinical practice are still limited in Korea.

RCTs are generally considered the gold standard for evaluating the efficacy of new therapeutic interventions for defined medical conditions. Standardized trial designs are necessary to ensure that patients randomly allocated to treatment arms are as similar as possible. The setting of RCT could therefore encompasses restrictive inclusion and exclusion criteria, fixed treatment regimens, and rigid follow-up protocol [13,14]. In rheumatology as in many other fields of medicine, clinical trials may not truly reflect the effectiveness of treatments in the real-world setting [15], particularly as most patients with the diagnosis of interest are ineligible for standard clinical trials $[16,17]$. In other words, the effectiveness of a new agent is also determined by how well it performs in real-world conditions, outside the context of a randomized trial [18]. The aim of this study was to analyze the effectiveness of bDMARDs and cDMARD treatment in daily clinical practice in Korea.

\section{METHODS}

\section{Patients}

The Korean multicenter observational study of disease activity changes in DMARD users with seropositive RA (KOSDAI) study was an observational study conducted in 24 secondary or tertiary medical institutes nationwide between January 2012 and April 2014. Seropositive patients aged $\geq 18$ years were required to meet the criteria of the 2010 American College of Rheumatology (ACR)/ European League Against Rheumatism (EULAR) for RA [19] and the following criteria: tender joint count (TJC) $\geq 3$, swollen joint count $(\mathrm{SJC}) \geq 2$, and C-reactive protein $(\mathrm{CRP}) \geq 1 \mathrm{mg} / \mathrm{dL}$ or erythrocyte sedimentation rate (ESR) $\geq 28 \mathrm{~mm} / \mathrm{hr}$. Patients were screened and enrolled at the time point of their regular visit to the outpatient clinic. Patients enrolled in the study had received a regimen that was deemed appropriate by their treating physician. The study design was in accordance with the ethical standards of the Institutional and/or National Research Committee and the Declaration of Helsinki (as revised in Brazil 2013). The study protocol was reviewed by Institutional Review Board at each site (SMG-SNU Boramae Medical center 20120229/06-2012-42/117, et al.). Informed consent was obtained from all participants in the study.

\section{Clinical and laboratory assessment}

Clinical and demographic characteristics were collected on case report forms at the time of enrollment. Clinical features were assessed at baseline and at month 6 and month 12. Simplified disease activity index (SDAI) score was employed to evaluate disease activity and remission. SDAI score was calculated as the sum of TJC, SJC, patient global assessment (PGA; $10 \mathrm{~cm}$ visual analogue scale [VAS]), and CRP level. SDAI score of $\leq 3.3$ indicated remission while a score of 3.3 to $11,>11$ to $\leq$ 26 , and $>26$ indicated low, moderate, and high disease activity, respectively. Physician global assessment (10 cm VAS) and Health Assessment Questionnaire (HAQ) scores were also obtained. To assess clinical remission, the 2011 ACR/EULAR remission criteria were used as the main tool [20]. Data on baseline rheumatoid factor and/or anti-cyclic citrullinated peptide antibody were 
obtained. CRP and ESR levels were measured at baseline and month 6 and month 12 .

\section{Statistical analyses}

Patients who initiated a bDMARD on enrollment or who started on a cDMARD but switched to a bDMARD during the 12-month period were included in the bDMARD group. Main analyses were conducted for the full analysis set. Those who were treated with a bDMARD or cDMARD for $\geq 6$ months were also analyzed. Statistical analyses were performed using SPSS statistical software version 22.0 (IBM Co., Armonk, NY, USA). SDAI scores were analyzed using Wilcoxon's rank sum test and Wilcoxon's signed rank test. Pearson's chi-square test was used to evaluate disease activity and remission between patients treated with DMARDs. A $p<0.05$ was considered statistically significant. Factors affecting remission were analyzed using a multivariate linear regression model with treatment group, sex, age, disease duration, and baseline SDAI score as independent variables and remission as dependent variable.

\section{RESULTS}

\section{Patient demographics}

Data of 802 seropositive RA patients were analyzed (Fig. 1). A total of 660 patients (82\%) completed follow-up for 12 months, including 317 patients in the cDMARD group and 343 patients in the bDMARD group. Patient demographics and clinical parameters at baseline, including SDAI and disease activity score 28 (DAS28) are summarized in Table 1. The mean age was 57.0 years in the cDMARD group and 54.2 years in the bDMARD group. Patients were predominantly women, with a disease duration of $5.9 \pm 6.6$ and $7.8 \pm 7.5$ years in cDMARD and bDMARD groups, respectively, showing significant $(p<0.0001)$ difference between the two groups. Baseline SDAI was higher in the bDMARD group than that in the cDMARD group $(32.08 \pm 12.98$ vs. $25.69 \pm 10.90$, $p<0.0001$ ). All patients in the bDMARD group were prescribed with cDMARDs for at least 6 months before starting bDMARDs following the Korean National Health Insurance reimbursement guidelines [21]. Biologic DMARDs mainly comprised of TNF- $\alpha$ antagonists (Supplementary Table 1). Sixteen percent of bDMARD

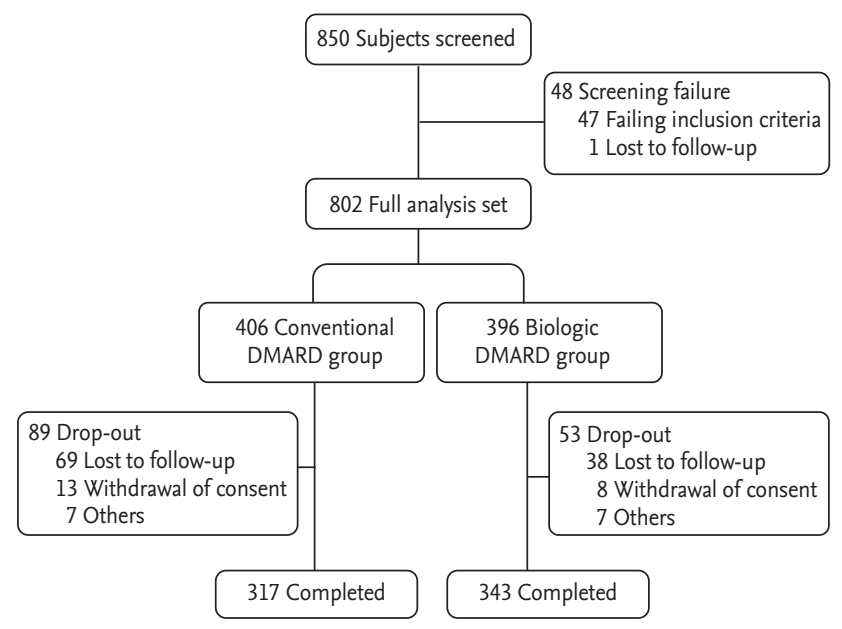

Figure 1. Study flow and patient disposition. DMARD, disease-modifying antirheumatic drug.

users were prescribed more than two different agents. Most patients (97.5\%) in the bDMARD group also received a cDMARD during the study period (Supplementary Table 1).

\section{Changes in disease activity scores}

Follow-up disease activity parameters were measured at 6 and 12 months. Fig. 2 shows changes in SDAI score in each group. SDAI was improved during the follow-up period. The mean SDAI in the bDMARD group was decreased from $32.08 \pm 12.98$ at baseline to $15.49 \pm 11.69$ at 6 months and $12.91 \pm 12.16$ at 12 months while the mean SDAI in the cDMARD group was decreased from 25.69 \pm 10.90 to $15.10 \pm 23.65$ at 6 months and $12.28 \pm 13.50$ at 12 months. Overall, the mean change in SDAI at 12 months was -19.0 in the bDMARD group and -12.6 in the cDMARD group (both $p<0.0001$ ). Most patients (64.2\%) had high SDAI in the bDMARD group while most patients $(58.3 \%, p<0.0001)$ had moderate SDAI in the cDMARD group (Fig. 3). Although baseline SDAI scores were higher in the bDMARD group, SDAI scores were rapidly decreased after receiving bDMARD, leading to no significant difference in SADI score between the two groups at 6 or 12 months.

Likewise, baseline disease activity parameters including TJC, SJC, PGA, DAS28, HAQ, and ESR were significantly higher in the bDMARD group (Table 2). After 6 months, there were no significant differences in TJC, SJC, or PGA between the two groups. DAS28 also be- 
Table 1. Baseline demographics and clinical characteristics of patient enrolled in this study

\begin{tabular}{|c|c|c|c|}
\hline Characteristic & $\begin{array}{l}\text { Conventional DMARD group } \\
\qquad(\mathrm{n}=406)\end{array}$ & $\begin{array}{l}\text { Biologic DMARD group } \\
\qquad(\mathrm{n}=396)\end{array}$ & $p$ value \\
\hline Age, yr & $57.0 \pm 12.2$ & $54.2 \pm 12.3$ & 0.0010 \\
\hline Female sex & $325(80.1)$ & $345(87.1)$ & 0.0069 \\
\hline Disease duration, yr & $5 \cdot 9 \pm 6.6$ & $7.8 \pm 7.5$ & $<0.0001$ \\
\hline Rheumatoid factor, IU/mL & $281.9 \pm 687.0$ & $215.2 \pm 324.1$ & 0.9845 \\
\hline Anti-CCP, IU/mL & $267.6 \pm 598.0$ & $262.2 \pm 524.6$ & 0.1300 \\
\hline $\mathrm{ESR}, \mathrm{mm} / \mathrm{hr}$ & $51.7 \pm 26.3$ & $58.6 \pm 26.0$ & 0.0002 \\
\hline $\mathrm{CRP}, \mathrm{mg} / \mathrm{dL}$ & $2.84 \pm 5 \cdot 51$ & $2.59 \pm 3.48$ & 0.4318 \\
\hline SDAI & $25.69 \pm 10.90$ & $32.08 \pm 12.98$ & $<0.0001$ \\
\hline DAS28-ESR & $4.70 \pm 0.71$ & $5.23 \pm 0.80$ & $<0.0001$ \\
\hline TJC, n (o-28) & $6.9 \pm 4.4$ & $10.2 \pm 6.1$ & $<0.0001$ \\
\hline SJC, n (o-28) & $5 \cdot 3 \pm 3 \cdot 5$ & $7 \cdot 4 \pm 5.0$ & $<0.0001$ \\
\hline PGA, cm (o-10) & $5 \cdot 3 \pm 2.1$ & $6.0 \pm 2.2$ & $<0.0001$ \\
\hline MDGA, cm (o-10) & $5 \cdot 4 \pm 2.1$ & $5 \cdot 9 \pm 2.1$ & 0.0020 \\
\hline HAQ & $1.00 \pm 0.73$ & $1.33 \pm 0.75$ & $<0.0001$ \\
\hline \multicolumn{4}{|l|}{ Previous DMARD use, \% } \\
\hline Conventional & 88.9 & 100 & - \\
\hline Biologic & 6.7 & 20.7 & - \\
\hline Corticosteroid use, \% & 92.1 & 85.9 & - \\
\hline
\end{tabular}

Values are presented as mean \pm SD or number (\%).

DMARD, disease-modifying antirheumatic drug; CCP, cyclic citrullinated peptide; ESR, erythrocyte sedimentation rate; CRP, C-reactive protein; SDAI, simplified disease activity index; DAS, disease activity score; TJC, tender joint count; SJC, swollen joint count; PGA, patient global assessment; MDGA, physician global assessment; HAQ, Health Assessment Questionnaire.

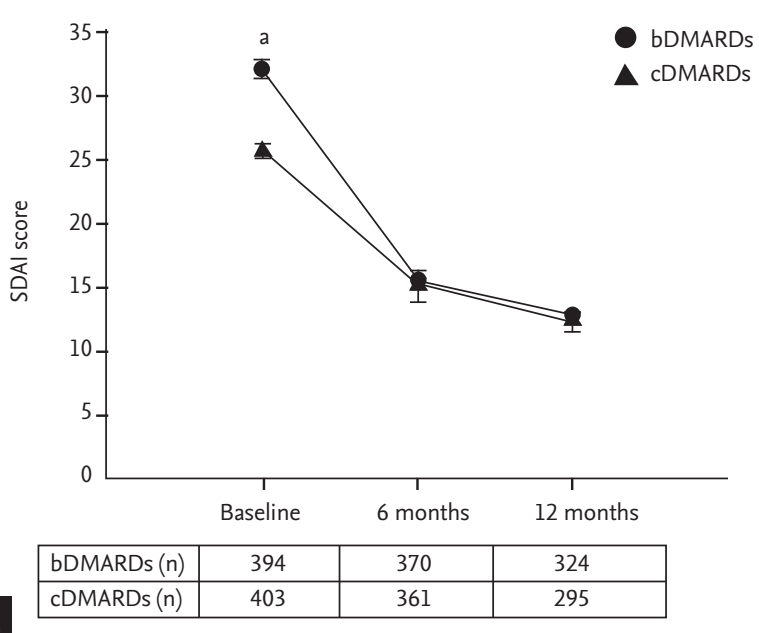

Figure 2. Reduction of simplified disease activity index (SDAI) after 6 and 12 months. (A) Total patient population irrespective of treatment period. (B) Patients who received conventional disease-modifying antirheumatic drug (cDMARD) or biologic DMARD (bDMARD) for at least 6 months. Data represent mean \pm standard error. ${ }^{\mathrm{a}} \mathrm{p}<0.0001$. 


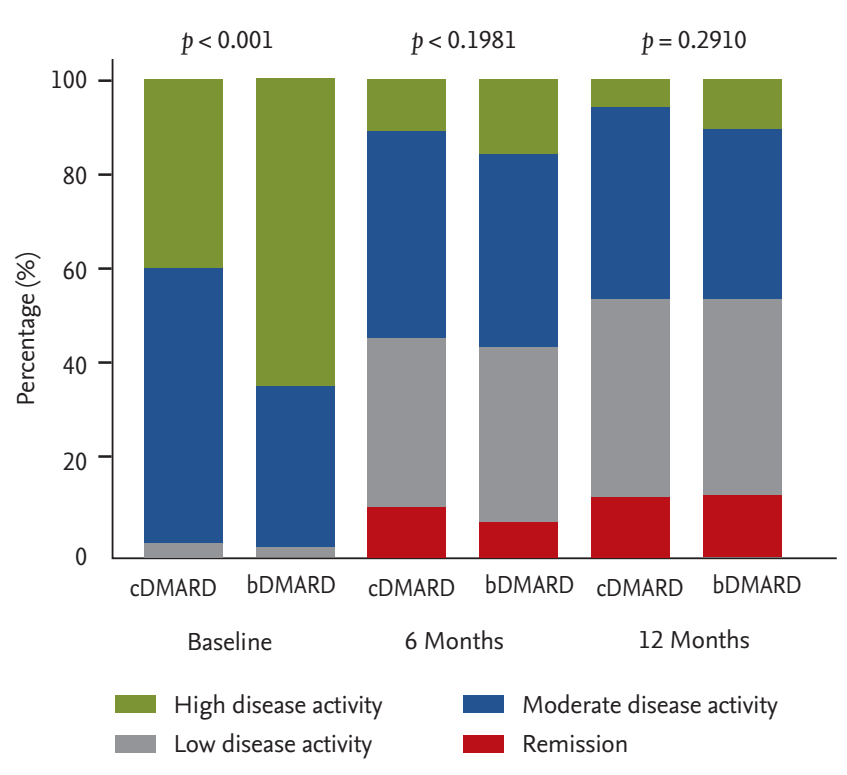

Figure 3. Changes in distribution of simplified disease activity index (SDAI) in conventional disease-modifying antirheumatic drug (cDMARD) or biologic DMARD (bDMARD) groups at 6 and 12 months. Depicted numbers are percentages of patients.

came comparable between the two groups at 12 months.

\section{Factors affecting clinical remission at 12 months}

Baseline disease activities were higher in the bDMARD group. Forty-eight patients (13.3\%) in the cDMARD group and 32 patients $(8.7 \%, p=0.0442)$ in the bDMARD group fulfilled the 2011 ACR/EULAR remission criteria at the 6-month follow-up visit (Table 3). However, at 12 months, the two groups showed no significant difference in remission rate (14.6\% vs. $15.4 \%)$. The remission rate in the bDMARD group increased notably in the latter 6-month period. Similar results were observed for SDAI values of remission (Fig. 3).

Results of univariate analysis of baseline variables associated with remission (2011 ACR/EULAR remission criteria at 12 months) are shown in Table 4. Baseline SDAI and HAQ scores were the best predictive factors for remission. However, treatment group, sex, age, or disease duration was not predictive of remission. In multivariate logistic regression analysis, baseline SDAI and HAQ scores were analyzed separately since both factors were markers of disease activities. Baseline HAQ score was the most significant factor associated with remission.

\section{DISCUSSION}

The KOSDAI study aimed to investigate 'real-world' changes in disease activity of moderate to highly active seropositive Korean RA patients visiting rheumatology clinics, especially those who required bDMARDs. Baseline SDAI score was higher in the bDMARD group. However, after 6 months of treatment, there were steep reductions in SDAI, resulting in no significant difference in SDAI between bDMARD- and cDMARD-treated patients. Similar trends were observed for other clinical and laboratory parameters. Different baseline characteristics such as disease activity, levels of inflammatory markers, and disease duration are somewhat expected in daily clinical practice because patients with greater disease severity would be preferentially prescribed with bDMARDs [22]. In our study, patients who received bDMARDs eventually showed significantly reduced disease activity, particularly those who were receiving bDMARDs for $\geq 6$ months (Fig. 2B).

It is noteworthy that patients in the cDMARD group also achieved significant reduction of disease activity in this observational study. This indicates that the aim of lowering disease activity is well pursued based on patient's clinical status or prognostic factors either using bDMARDs or single/combination use of cDMARDs (Supplementary Table 1). Surprisingly, the clinical remission rate in the cDMARD group at 6 months was greater than that in patients treated with bDMARD. This is in part because of the higher baseline SDAI in the bDMARD group. In addition, $12 \%$ of patients in the cDMARD group were DMARD-naïve (Table 1). Nevertheless, at 12 months, the percentage of patients achieving remission in the bDMARD group exceeded that in the cDMARD group.

In our study, baseline HAQ was identified as a key predictor of remission. This corroborates with previous studies of bDMARDs on key factors associated with remission, showing that patients with low HAQ or DASs have better clinical outcomes $[23,24]$. In contrast, treatment group, sex, age, or disease duration did not affect clinical remission in our study. A study from the British Society for Rheumatology Biologics Register showed that females were less likely to achieve remission [23]. In our study, higher percentage of females in the bDMARD group along with higher SDAI at baseline might have con- 
Table 2. Changes in clinical and laboratory parameters

\begin{tabular}{|c|c|c|c|c|}
\hline Parameter & Visit & $\begin{array}{l}\text { Conventional DMARD group } \\
\qquad(\mathrm{n}=406)\end{array}$ & $\begin{array}{l}\text { Biologic DMARD group } \\
(\mathrm{n}=396)\end{array}$ & $p$ value \\
\hline \multirow[t]{3}{*}{ TJC, n (o-28) } & Baseline & $6.9 \pm 4.4$ & $10.2 \pm 6.1$ & $<0.0001$ \\
\hline & 6 mon & $3.4 \pm 4.0$ & $4.2 \pm 4.8$ & 0.0589 \\
\hline & 12 mon & $2.6 \pm 3.7$ & $2.9 \pm 4.1$ & 0.9262 \\
\hline \multirow[t]{3}{*}{ SJC, n (o-28) } & Baseline & $5 \cdot 3 \pm 3 \cdot 5$ & $7 \cdot 4 \pm 5.0$ & $<0.0001$ \\
\hline & 6 mon & $2.1 \pm 2.6$ & $2.5 \pm 3.3$ & 0.3655 \\
\hline & 12 mon & $1.6 \pm 2.4$ & $2.0 \pm 3.0$ & 0.2038 \\
\hline \multirow[t]{3}{*}{ CRP, mg/dL } & Baseline & $2.84 \pm 5.51$ & $2.59 \pm 3.48$ & 0.2168 \\
\hline & 6 mon & $2.49 \pm 22.17$ & $1.36 \pm 3.88$ & 0.2189 \\
\hline & 12 mon & $1.65 \pm 9.97$ & $1.53 \pm 6.95$ & 0.7348 \\
\hline \multirow[t]{3}{*}{$\mathrm{ESR}, \mathrm{mm} / \mathrm{hr}$} & Baseline & $51.7 \pm 26.3$ & $58.6 \pm 26.0$ & 0.0002 \\
\hline & 6 mon & $34.0 \pm 23.7$ & $41.3 \pm 28.4$ & 0.0002 \\
\hline & 12 mon & $33.2 \pm 23.6$ & $39.0 \pm 27.4$ & 0.0048 \\
\hline \multirow[t]{3}{*}{ DAS28-ESR } & Baseline & $4.70 \pm 0.71$ & $5.23 \pm 0.80$ & $<0.0001$ \\
\hline & 6 mon & $3.42 \pm 1.09$ & $3.70 \pm 1.16$ & 0.0012 \\
\hline & 12 mon & $3.22 \pm 1.03$ & $3.38 \pm 1.16$ & 0.0609 \\
\hline \multirow[t]{3}{*}{ PGA, cm (o-10) } & Baseline & $5 \cdot 3 \pm 2.1$ & $6.0 \pm 2.2$ & $<0.0001$ \\
\hline & 6 mon & $3.6 \pm 2.3$ & $3.9 \pm 2.4$ & 0.1615 \\
\hline & 12 mon & $3 \cdot 3 \pm 2.2$ & $3.4 \pm 2.4$ & 0.7670 \\
\hline \multirow[t]{3}{*}{ HAQ } & Baseline & $1.00 \pm 0.73$ & $1.33 \pm 0.75$ & $<0.0001$ \\
\hline & 6 mon & $0.66 \pm 0.63$ & $0.91 \pm 0.70$ & $<0.0001$ \\
\hline & 12 mon & $0.61 \pm 0.63$ & $0.85 \pm 0.71$ & $<0.0001$ \\
\hline
\end{tabular}

Values are presented as mean $\pm \mathrm{SD}$.

DMARD, disease-modifying antirheumatic drug; TJC, tender joint count; SJC, swollen joint count; CRP, C-reactive protein; ESR, erythrocyte sedimentation rate; DAS, disease activity score; PGA, patient global assessment; HAQ, Health Assessment Questionnaire.

Table 3. Clinical remission rate according to the 2011 ACR/EULAR remission criteria

\begin{tabular}{|c|c|c|c|c|}
\hline Visit & Remission & Conventional DMARD group & Biologic DMARD group & $p$ value \\
\hline \multirow[t]{2}{*}{6 mon } & Yes & $48(13.30)$ & $32(8.65)$ & 0.0442 \\
\hline & No & $313(86.70)$ & 338 (91.35) & \\
\hline \multirow[t]{2}{*}{12 mon } & Yes & $43\left(14.5^{8}\right)$ & $50(15 \cdot 43)$ & 0.7660 \\
\hline & No & $252(85.42)$ & $274(84.57)$ & \\
\hline
\end{tabular}

Values are presented as number (\%).

ACR, American College of Rheumatology; EULAR, European League against Rheumatism; DMARD, disease-modifying antirheumatic drug.

tributed to the observed rate of remission at 6 months.

Characteristics of the bDMARD group were confounded by indication. Age, disease duration, disease activity, and inflammatory marker levels at baseline all reflected clinical status of study patients at enrollment. Thus, there could be a selection bias which limits fur- 
Table 4. Factors affecting clinical remission in study patients

\begin{tabular}{|c|c|c|c|c|c|c|}
\hline \multirow{2}{*}{ Variable } & \multicolumn{2}{|c|}{ Univariate analysis } & \multicolumn{4}{|c|}{ Multivariate analysis } \\
\hline & OR (95\% CI) & $p$ value & Estimate & $\mathrm{SE}$ & OR (95\% CI) & $p$ value \\
\hline Treatment group $^{\mathrm{a}}$ & $1.078(0.693-1.677)$ & 0.7393 & & & & \\
\hline $\operatorname{Sex}{ }^{b}$ & $1.039(0.579-1.866)$ & 0.8972 & & & & \\
\hline Age & $0.988(0.970-1.006)$ & 0.1756 & & & & \\
\hline Disease duration & $0.999(0.996-1.001)$ & 0.3000 & & & & \\
\hline SDAI at baseline & $0.977(0.957-0.997)$ & $0.0251^{c}$ & & & & \\
\hline HAQ score at baseline & $0.662(0.483-0.907)$ & $0.0102^{c}$ & -0.435 & 0.165 & $0.648(0.469-0.894)$ & $0.0083^{d}$ \\
\hline
\end{tabular}

OR, odds ratio; CI, confidence interval; SE, standard error; SDAI, simplified disease activity index; HAQ, Health Assessment Questionnaire.

${ }^{\mathrm{a}} \mathrm{O}=$ conventional disease-modifying antirheumatic drug (DMARD), $1=$ biologic DMARD.

$\mathrm{b}_{\mathrm{O}}=$ female, $1=$ male.

${ }^{c} p<0.05$.

${ }^{\mathrm{d}} \mathrm{p}<0.01$.

ther comparison of SDAI changes with the cDMARD group. In addition, heterogeneity regarding previous DMARD use, previous or current biologic use, and concomitant DMARD prescriptions in the bDMARD group make it difficult to compare results between treatment groups. Moreover, a higher rate of drop out (21.9\%) in the cDMARD group might have resulted in reasonable outcomes seen in our study. Conversely, the lower rate of dropouts in the bDMARD group (13.3\%) may indicate improved adherence to bDMARDs over cDMARDs in RA patients with moderate to high disease activity, a finding that has also been reported previously [25]. However, it was impossible to clearly interpret whether dropout rates were due to the lack of effectiveness or specific adverse events in either group in our study.

Despite these potential limitations, this observational study collected clinical data from patients receiving standard RA treatment in daily clinical practice. Observational studies are of great value for obtaining data on effectiveness and prognosis in RA patients [26]. As few published data comparing DMARD use in Korean RA patients are available, we believe that this study has value in that it assesses a large number of RA patients, including analyzing their clinical outcomes after bDMARD use.

In conclusion, Korean seropositive RA patients with moderate to high disease activity irrespective of receiving cDMARDs or bDMARDs are being offered proper standardized care, showing marked decrease in SDAI at 6 months with additional reduction in the following 6 months. Low baseline HAQ was the best predictor for achieving remission at 1 year. Thus, those with higher HAQ at baseline would need further intervention to achieve better outcomes in longer term.

\section{KEY MESSAGE}

1. There was a significant reduction in simplified disease activity index within 12 months in Korean seropositive rheumatoid arthritis patients irrespective of biologic or conventional disease-modifying antirheumatic drug use in real-word practice.

2. Clinical remission was achieved particularly for those with lower baseline Health Assessment Questionnaire scores.

\section{Conflict of interest}

This work was supported by Eisai Korea Inc.

\section{Acknowledgments}

We would like to thank Hee Yeon Kay for providing help for the writing of this manuscript.

\section{REFERENCES}

1. Scott DL, Symmons DP, Coulton BL, Popert AJ. Long- 
term outcome of treating rheumatoid arthritis: results after 20 years. Lancet 1987;1:1108-1111.

2. Isomaki H. Long-term outcome of rheumatoid arthritis. Scand J Rheumatol Suppl 1992;95:3-8.

3. Sung YK, Cho SK, Choi CB, Bae SC. Prevalence and incidence of rheumatoid arthritis in South Korea. Rheumatol Int 2013;33:1525-1532.

4. Pressman Lovinger S. Use of biologics for rheumatoid arthritis tempered by concerns over safety, cost. JAMA 2003;289:3229-3230.

5. Scott DL, Kingsley GH. Tumor necrosis factor inhibitors for rheumatoid arthritis. N Engl J Med 2006;355:704-712.

6. Maini RN, Breedveld FC, Kalden JR, et al. Therapeutic efficacy of multiple intravenous infusions of anti-tumor necrosis factor alpha monoclonal antibody combined with low-dose weekly methotrexate in rheumatoid arthritis. Arthritis Rheum 1998;41:1552-1563.

7. Weinblatt ME, Keystone EC, Furst DE, et al. Adalimumab, a fully human anti-tumor necrosis factor alpha monoclonal antibody, for the treatment of rheumatoid arthritis in patients taking concomitant methotrexate: the ARMADA trial. Arthritis Rheum 2003;48:35-45.

8. Keystone EC, Schiff MH, Kremer JM, et al. Once-weekly administration of $50 \mathrm{mg}$ etanercept in patients with active rheumatoid arthritis: results of a multicenter, randomized, double-blind, placebo-controlled trial. Arthritis Rheum 2004;50:353-363.

9. Keystone EC, Genovese MC, Klareskog L, et al. Golimumab, a human antibody to tumour necrosis factor \{alpha\} given by monthly subcutaneous injections, in active rheumatoid arthritis despite methotrexate therapy: the GO-FORWARD Study. Ann Rheum Dis 2009;68:789-796.

10. Maini R, St Clair EW, Breedveld F, et al. Infliximab (chimeric anti-tumour necrosis factor alpha monoclonal antibody) versus placebo in rheumatoid arthritis patients receiving concomitant methotrexate: a randomised phase III trial. ATTRACT Study Group. Lancet 1999;354:19321939.

11. McKeage K. A review of CT-P13: an infliximab biosimilar. BioDrugs 2014;28:313-321.

12. Ravindran V, Scott DL, Choy EH. A systematic review and meta-analysis of efficacy and toxicity of disease modifying anti-rheumatic drugs and biological agents for psoriatic arthritis. Ann Rheum Dis 2008;67:855-859.

13. Furst DE. Observational cohort studies and well controlled clinical trials: we need them both! J Rheumatol
2004;31:1476-1477.

14. Pincus T, Sokka T. Should contemporary rheumatoid arthritis clinical trials be more like standard patient care and vice versa? Ann Rheum Dis 2004;63 Suppl 2:ii32-ii39.

15. Krishnan E, Fries JF. Measuring effectiveness of drugs in observational databanks: promises and perils. Arthritis Res Ther 2004;6:41-44.

16. Sokka T, Pincus T. Eligibility of patients in routine care for major clinical trials of anti-tumor necrosis factor alpha agents in rheumatoid arthritis. Arthritis Rheum 2003;48:313-318.

17. Sokka T, Pincus T. Most patients receiving routine care for rheumatoid arthritis in 2001 did not meet inclusion criteria for most recent clinical trials or American College of Rheumatology criteria for remission. J Rheumatol 2003;30:1138-1146.

18. Bombardier C, Maetzel A. Pharmacoeconomic evaluation of new treatments: efficacy versus effectiveness studies? Ann Rheum Dis 1999;58 Suppl 1:I82-I85.

19. Aletaha D, Neogi T, Silman AJ, et al. 2010 Rheumatoid arthritis classification criteria: an American College of Rheumatology/European League Against Rheumatism collaborative initiative. Arthritis Rheum 2010;62:25692581.

20. Felson DT, Smolen JS, Wells G, et al. American College of Rheumatology/European League Against Rheumatism provisional definition of remission in rheumatoid arthritis for clinical trials. Arthritis Rheum 2011;63:573-586.

21. Hur JW, Choe JY, Kim DW, et al. Rheumatoid arthritis patients fulfilling Korean National Health Insurance reimbursement guidelines for anti-tumor necrosis factor- $\alpha$ treatment and comparison to other guidelines. Rheumatol Int 2015;35:1817-1823.

22. Singh JA, Saag KG, Bridges SL Jr, et al. 2015 American College of Rheumatology guideline for the treatment of rheumatoid arthritis. Arthritis Rheumatol 2016;68:1-26.

23. Hyrich KL, Watson KD, Silman AJ, Symmons DP; British Society for Rheumatology Biologics Register. Predictors of response to anti-TNF-alpha therapy among patients with rheumatoid arthritis: results from the British Society for Rheumatology Biologics Register. Rheumatology (Oxford) 2006;45:1558-1565.

24. Anderson JJ, Wells G, Verhoeven AC, Felson DT. Factors predicting response to treatment in rheumatoid arthritis: the importance of disease duration. Arthritis Rheum 2000;43:22-29. 
25. Zink A, Listing J, Kary S, et al. Treatment continuation in patients receiving biological agents or conventional DMARD therapy. Ann Rheum Dis 2005;64:1274-1279.

26. Hetland ML, Christensen IJ, Tarp U, et al. Direct comparison of treatment responses, remission rates, and drug adherence in patients with rheumatoid arthritis treated with adalimumab, etanercept, or infliximab: results from eight years of surveillance of clinical practice in the nationwide Danish DANBIO registry. Arthritis Rheum 2010;62:22-32. 
Supplementary Table 1. DMARDs used during the study period

\begin{tabular}{|c|c|c|}
\hline Variable & $\begin{array}{l}\text { Conventional DMARD group } \\
\qquad(\mathrm{n}=406)\end{array}$ & $\begin{array}{l}\text { Biologic DMARD group } \\
\qquad(\mathrm{n}=396)\end{array}$ \\
\hline Conventional DMARDs & $406(100.0)$ & $386(97.5)$ \\
\hline Methotrexate & $327(80.5)$ & $362(91.4)$ \\
\hline Hydroxychloroquine & $226(55 \cdot 7)$ & $62(15 \cdot 7)$ \\
\hline Leflunomide & $174(42.9)$ & $47(11.9)$ \\
\hline Sulfasalazine & $72(17.7)$ & $28(7.1)$ \\
\hline Tacrolimus & $55(13.6)$ & $18(4 \cdot 6)$ \\
\hline Bucillamine & $23(5 \cdot 7)$ & $6(1.5)$ \\
\hline Mizoribine & $8(2.0)$ & $5(1.3)$ \\
\hline Cyclosporine & $3(0.7)$ & $2(0.5)$ \\
\hline Azathioprine & $2(0.5)$ & - \\
\hline Biologic DMARDs & - & $396(100.0)$ \\
\hline Adalimumab & - & $212(53 \cdot 6)$ \\
\hline Etanercept & - & $130(32.8)$ \\
\hline Infliximab & - & $58(14.7)$ \\
\hline Rituximab & - & $33(8.3)$ \\
\hline Abatacept & - & $23(5.8)$ \\
\hline Tocilizumab & - & $7(1.8)$ \\
\hline Golimumab & - & $2(0.5)$ \\
\hline Secukinumab & - & $1(0.3)$ \\
\hline
\end{tabular}

Values are presented as number (\%). The number of agents used by each patient during the study period was counted. DMARD, disease-modifying antirheumatic drug. 